\title{
Early Visual Functional Outcomes and Morphological Responses to Anti-Vascular Growth Factor Therapy in Diabetic Macular Oedema Using Optical Coherence Tomography Angiography
}

This article was published in the following Dove Press journal: Clinical Ophthalmology

\section{Supriya Dabir iD ${ }^{\prime}$ \\ Mohan Rajan' \\ Liji Parasseril' \\ Vaidehi Bhatt ${ }^{2}$ \\ Preetam Samant ${ }^{3}$ \\ CAB Webers ${ }^{4}$ \\ TTJM Berendschot (iD ${ }^{4}$ \\ 'Department of Retina, Rajan Eye Care Pvt Ltd, Chennai, India; ${ }^{2}$ Rajiv Gandhi Medical College, Thane, India; ${ }^{3}$ Department of Retina, PD Hinduja Hospital and Medical Research Center, Mumbai, India; ${ }^{4}$ University Eye Clinic, Maastricht, the Netherlands}

Correspondence: Supriya Dabir

Rajan Eye Care Hospital Pvt. Ltd, No. 5, Vidyodaya East 2nd Street, T. Nagar,

Chennai, Tamil Nadu 6000I7, India

Tel +919900974800

Email supriad@gmail.com
Purpose: Diabetic macular oedema (DME) is a vision-threatening complication of diabetic retinopathy. Spectral domain optical coherence tomography angiography (OCTA) is useful for assessing DME. We performed serial OCTA measurements before and after 3 monthly intravitreal anti-vascular endothelial growth factor (anti-VEGF) injections to understand its relationship with best corrected visual acuity (BCVA), central macular thickness (CMT) and vascular indices. Methods: OCTA assessment consisting of $6 \times 6 \mathrm{~mm}$ scans centred on fovea and en-face retinal angiograms were obtained. Scans were done at baseline and at 1 month following each of the 3 intravitreal injections of anti-VEGF (ranibizumab, Lucentis ${ }^{\circledR}$ ).

Results: Our prospective study included 24 eyes of 24 subjects, aged $63.0 \pm 5.0$ years. There was a mean gain of $0.07 \operatorname{LogMAR}(3$ letters) and reduction of CMT, vessel density (VD) and perfusion density (PD) by end of 3rd month. CMT was independently associated with improvement in BCVA. There were significant reductions in FAZ area, VD and PD over 3 months from baseline.

Conclusion: At three months, intravitreal anti-VEGF therapy is associated with visual function recovery in DME, with reduction in CMT being the most reliable predictor of response. OCTA shows a reduction in VD and PD respectively suggestive of reduced macular perfusion in the short term. Simultaneous reduction in FAZ size can be due to reversal of manual displacement of the retinal capillaries resulting from resolution of intraretinal oedema.

Keywords: diabetic macular oedema, superficial capillary plexus, vascular density, perfusion density, foveal avascular zone, optical coherence tomography angiography, macular ischemia

\section{Introduction}

Diabetic retinopathy (DR) is a common cause of acquired loss of vision in adults worldwide and is known to be a progressive microvascular complication of diabetes mellitus. India alone is estimated to have 72 million people with diabetes mellitus (DM), and potentially $33 \%$ of them are at risk of developing retinopathy. ${ }^{1}$ With a prevalence and a rising trend in diagnosis of DM at younger age, the incidence of retinopathy is expected to rise. In fact, the risk of developing sight-threatening DR is 2 times higher in people who develop DM earlier in life ( $<40$ years) and nearly 6.5 times higher in individuals with long-standing DM ( $>16$ years). ${ }^{2}$ 
Diabetic macular oedema (DME) is the most common vision-threatening complication of DR and is estimated to be affecting $\sim 9 \%$ of all patients with any form of DR in India. ${ }^{1}$ A metabolic cascade secondary to systemic hyperglycaemia results in the disruption of the blood-retinal barrier (BRB) and is a consequent cause of macular oedema. Although inflammatory dysregulation of the vascular endothelium could be a critical pathway, it is also found that the swelling and subsequent apoptosis of pericytes activates the cascade of vasoconstriction, followed by disruption resulting in vasculopathy and fluid collection. ${ }^{3,4}$ Hypoxia caused by the microvascular disease stimulates the release of vascular endothelial growth factor (VEGF), leading to increased vascular permeability and resultant retinal oedema. ${ }^{3}$ In recent years, the use of intravitreal anti-VEGF injections has become the standard of care in managing diabetic macular oedema. ${ }^{5}$ Intravitreal steroids are used as an alternative in patients who fail to respond to anti-VEGF, elderly individuals with pseudophakia or when they are unable to take monthly injections. These therapies are also best used with routine monitoring and measurements of the retinal vasculature as frequently as possible to obtain safe and effective patient outcomes. While fluorescein angiography had been the established gold standard for assessing retinal vasculature, it is limited by its invasiveness, use of contrast and limited ability to obtain quantifiable measurements of avascular area and vessel density. ${ }^{6}$

Newer non-invasive modalities such as spectral domain optical coherence tomography (SD-OCT) have paved a way for assessing the effectiveness of intravitreal antiVEGF treatment in patients with DME and are the current gold standards in care. ${ }^{6}$ Spectral domain optical coherence tomography angiography (OCTA) has added a new dimension to understanding the disease as it gives the vascular pattern of the different layers of the retinal capillaries. It was limited in its use till lately because of being predominantly qualitative and time-consuming and because of lack of an applicable software. Now with the automated software built into the machine, it is easier to quantify the vascular features. Studies in the past have described the various changes that occur on OCT in diabetic retinopathy. However, more studies are needed in the Indian setting to understand the relationship between the OCTA markers and improvement in the visual acuity following antiVEGF therapy, given the high burden of cases and resource-poor setting so as to tailor optimal strategies. To understand the recovery in retinal microvasculature and treatment responses to anti-VEGF agents, in this study our objective was to perform serial OCTA measurements before and after monthly intravitreal anti-VEGF injections (ranibizumab, Lucentis ${ }^{\circledR}$ ). Our aim was to understand the changes in central macular thickness (CMT), vascular indices and foveal avascular zone (FAZ). We also explored the relationship between the change in the best corrected visual acuity (BCVA) with OCTA measures including CMT, FAZ, vessel density (VD) and perfusion density (PD).

\section{Patients and Methods}

This prospective study at Rajan Eye Care Hospital Pvt Limited, a tertiary eye care centre in Chennai, South India, included patients with type 2 diabetes who were diagnosed with diabetic macular oedema, defined as central retinal thickness greater than $300 \mathrm{~mm}$. The clinical severity of DR in each patient was classified using the International Clinical Diabetic Retinopathy Disease Severity Scale. Eyes with moderate-severe DR were included in this study. We included both treatment-naïve eyes and eyes that had received previous anti-VEGF or focal photocoagulation treatments more than 6 months before the base-line. We excluded patients with retinal vascular diseases, uveitis, glaucoma. Any media opacities like dense cataracts and patients with OCTA scans with signal strength less than 6 were not enlisted in the study. Patients with systemic history of recent cerebrovascular accident/ischemic heart disease in the last 6 months were also excluded. The methodology adhered to the tenets of the Declaration of Helsinki and the Rajan eye care ethical Committee. Informed consent was obtained from all patients.

All patients underwent a comprehensive ophthalmic evaluation including SD-OCT, OCTA, best corrected visual acuity (BCVA), tonometry, slit lamp bio microscopy and indirect fundus examination at baseline, 1 month, 2 months and 3 months. The patients received loading monthly injections for a period of three months irrespective of the fluid status on SD-OCT (fixed dosage regimen). Physician clearance was obtained if the patient had a recent history of systemic thromboembolic event within the past three months. One-week variation in dosing was allowed. The procedure was done by an experienced retina surgeon in the Operation Theatre, under aseptic precautions. OCTA assessment was done using the Zeiss Angioplex - 5000 OCTA system (Carl Zeiss Meditec, USA). The scans were acquired by a technician well 
trained in obtaining OCTA. Only scans with signal strength more than 6 were considered. A $6 \times 6 \mathrm{~mm}$ scan centred on the fovea was acquired, and en-face retinal angiograms were created using the proprietary Optical Micro Angiography (OMAGC) algorithms from the manufacturer that utilize amplitude and phase OCT signal data to deliver the angiography images. After acquisition, automated measurements from the superficial capillary plexus were used for study purposes. Parameters used were central macular thickness, perfusion density, vascular density and the size of the foveal avascular zone (FAZ). The scan was done 4 times in total for each patient, at baseline and at 1 month after each of the 3 injections.

\section{Statistical Analysis}

Statistical calculations were done with SPSS statistical software (version 25, IBM Corp, Armonk, USA). To study changes in time we used a general linear model repeated analysis and performed a linear mixed model analysis. Results are expressed as mean \pm standard deviation. To analyse visual acuity, we used LogMAR values. A $p$ value of $<0.05$ was considered significant.

\section{Results}

In this study we included 24 eyes of 24 subjects ( 9 female, 15 male), aged $63.0 \pm 5.0$ years and of which 9 were pseudophakic. Eleven patients had moderate NPDR and 13 severe NPDR with a mean duration of diabetes mellitus around $16.2 \pm 4.0$ years. Twenty-one eyes were treatmentnaïve, while 3 eyes had previously received treatment with anti-VEGF injections \pm laser, minimum 6 months prior to the study. We treated 14 right eyes and 10 left eyes.

Visual acuity improved in time. Vessel density and PD decreased significantly in time as seen in Figure 1 for all four areas as well as the area and perimeter of the FAZ and the CMT. We found no differences in time in the circularity of the FAZ (Table 1).

We performed a Pearson correlation between LogMAR, CMT, area and perimeter of the FAZ on one hand and VD and PD on the other hand (Table 2). It showed that the vessel and perfusion densities at the different areas all correlated with each other significantly $(r>0.97, p<0.001)$. Thus, a decrease in VD and PD was significantly correlated with reduction in CMT and decrease in area and perimeter of FAZ. Since VD and PD correlated with CMT, area and perimeter of the FAZ, which changed over time, the observed decreases in VD and PD may just be attributable to the decrease in the three last-mentioned. We therefore performed a linear mixed model analysis with eye as grouping factor, the vessel or perfusion density as the dependent variable and time, central macular thickness and area and perimeter of the fovea avascular zone as covariates (Table 3). It showed that the central macular thickness and the area of the avascular zone independently contributed to the vessel and perfusion density changes. To be precise, a decrease in the CMT and FAZ size, respectively, was positively correlated with a reduction in VD and $\mathrm{PD}$, respectively.

The perimeter of the foveal avascular zone did not contribute additionally, nor did we find an additional independent change in time. For LogMAR, central macular thickness appeared to be the only predictor in this approach.

\section{Discussion}

The current study aimed at measuring longitudinal changes in the functional (BCVA) and anatomical markers following 3 monthly intravitreal anti-VEGF injections in patients with moderate to severe NPDR, and DME based on OCTA. Early responses to anti-VEGF injections reflected a significant visual function recovery as noted by a mean gain of 0.7 LogMAR (3 letters) within the group, as well as the reduction of the central CMT, VD and PD by the end of the 3rd month. Most of the individuals can be considered to have responded to the antiVEGF in terms of resolution of macular oedema, and the average reduction of CMT observed was $\sim 120 \mu \mathrm{m}$ at the end of the $3 \mathrm{rd}$ month (Table 1$){ }^{7}$ A similar reduction in the CMT has been observed in past studies as well. ${ }^{8,9}$ Although individual variability did exist in the extent of response amongst the individuals, given the small numbers we were unable to perform any sub-group analysis to explore the potential predictors of good and poor response. Past studies also indicated significant variability in the resolution of macular oedema to anti-VEGF therapy, and up to $50 \%$ of the treated individuals have been noted to show significant reduction, and, at times, the remainder of them respond poorly to the anti-VEGF therapy. ${ }^{10}$

The analyses also highlighted that the reduction in the CMT was independently associated and predicted the improvement in the BCVA (Table 3). Indeed, oedema reduction and subsequent neural activation could promote synergistically the recovery in the microvascular dysfunction too. Previous studies indicated persistent macular oedema to be a negative prognostic factor in achieving desired visual acuity both in the short and long term. ${ }^{9,11}$ 


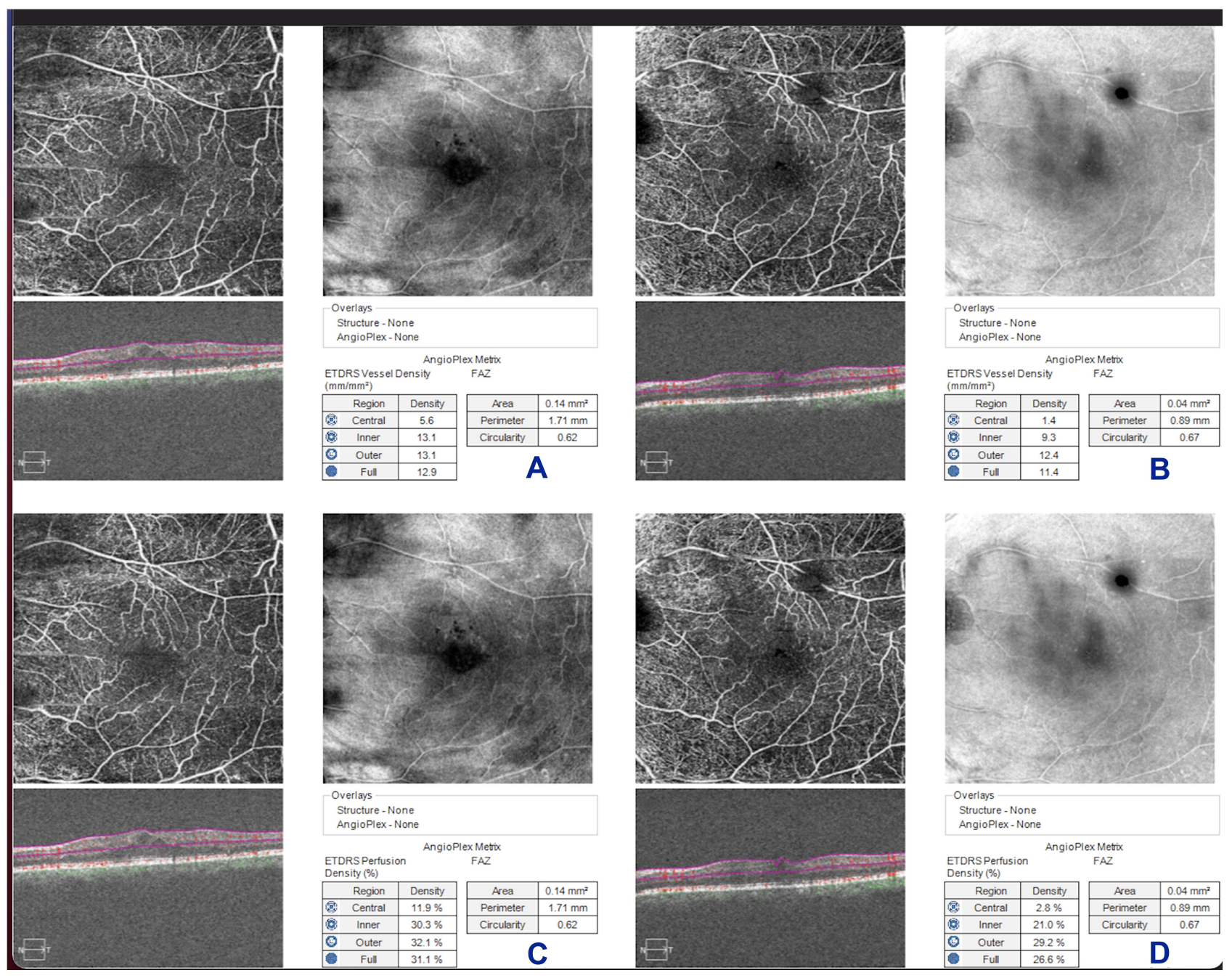

Figure I Shows the SCP with the vessel density and perfusion density at baseline and I month after the 3rd injection of a study patient. It shows the superficial capillary plexus: (A) with vessel density calculated at baseline; (B) with vessel density calculated at the end of 3 months (after 3 consecutive injections of anti-VEGF); (C) with perfusion density calculated at baseline; (D) with perfusion density calculated at the end of 3 months (after 3 consecutive injections of anti-VEGF).

Additionally, the study also demonstrates a significant reduction in the FAZ area and the VD and PD measures, respectively, over 3 months from baseline (Table 1).

We found that the FAZ area correlated with the reduction in the CMT; however, the model did not attribute any significant contribution of it in the apparent improvement in BCVA (Table 3). With the advent of OCTA and automated analyses, FAZ estimation has been noted to be reliable, even in comparison to the gold standard fluorescein angiography. ${ }^{12}$ Other studies have reported a strong negative correlation between FAZ size and visual acuity, and a larger FAZ size to be associated with poor BCVA. ${ }^{13}$ Reduction in FAZ following anti-VEGF therapies has been reflective of improved outcomes, and in fact non-reduction has been reported to be a predictor of recurrence of oedema. ${ }^{9,14}$ Anti-VEGF agents modulate vasculogenesis in the FAZ and support the optimization of optical path in the cone dense mosaic of the macula, resulting in improved retinal sensitivity. ${ }^{15,17}$

OCTA, unlike the dye-based angiography methods, allows one to quantify the retinal and choroidal vasculature non-invasively, based on the principle of measuring the scattered signals by the moving erythrocytes within the vessels. The VD is one such feature of interest and demonstrably differentiates between healthy eyes and eyes with diabetic retinopathy. Eyes with DR particularly are noted to have a lower VD in the superficial retinal layer as compared to their healthy matches. ${ }^{18,19}$ Also, most studies indicated the VD to be lower in eyes with macular oedema than in those without and baseline VD to be a predictor of good treatment response. ${ }^{8,14,20-22}$ In the study reported by AttaAllah 
Table I Visual Acuity, Central Macular Thickness and Different OCTA Parameters at Baseline and Follow-Ups

\begin{tabular}{|c|c|c|c|c|c|c|}
\hline & Baseline & I Month & 2 Months & 3 Months & $p^{a}$ & $p^{b}$ \\
\hline LogMAR & $0.27 \pm 0.18$ & $0.25 \pm 0.12$ & $0.25 \pm 0.18$ & $0.20 \pm 0.17$ & $0.017^{*}$ & $0.028^{*}$ \\
\hline Snellen ${ }^{c}$ & $0.54 \pm 0.22(20 / 37)$ & $0.56 \pm 0.16(20 / 36)$ & $0.56 \pm 0.23(20 / 36)$ & $0.63 \pm 0.25(20 / 32)$ & & \\
\hline CMT & $450 \pm 94$ & $409 \pm 81$ & $382 \pm 81$ & $331 \pm 69$ & $<0.001 *$ & $<0.001 *$ \\
\hline VD Central & $7.0 \pm 3.1$ & $5.4 \pm 2.4$ & $5.2 \pm 2.9$ & $4.0 \pm 2.3$ & $<0.001 *$ & $<0.001 *$ \\
\hline VD Inner & $13.2 \pm 1.8$ & $11.9 \pm 2.1$ & $10.8 \pm 3.4$ & $9.7 \pm 2.4$ & $<0.001 *$ & $<0.001 *$ \\
\hline VD Outer & $13.5 \pm 2.1$ & $12.2 \pm 1.8$ & $\mathrm{II} . \mathrm{I} \pm 2.4$ & $10.4 \pm 2.2$ & $<0.00 I^{*}$ & $<0.001 *$ \\
\hline VD Full & $13.2 \pm 1.9$ & $11.9 \pm 1.7$ & $10.9 \pm 2.6$ & $10.1 \pm 2.0$ & $<0.001 *$ & $<0.001 *$ \\
\hline PD Central & $14.8 \pm 7.2$ & $11.3 \pm 6.4$ & $11.2 \pm 6.7$ & $9.1 \pm 5.7$ & $<0.001 *$ & $0.00 I^{*}$ \\
\hline PD Inner & $31.0 \pm 4.3$ & $27.5 \pm 5.5$ & $25.6 \pm 8.8$ & $23.7 \pm 6.5$ & $<0.001 *$ & $<0.001 *$ \\
\hline PD Outer & $32.2 \pm 4.7$ & $29.3 \pm 5.0$ & $26.9 \pm 6.5$ & $26.5 \pm 5.4$ & $<0.001 *$ & $0.001 *$ \\
\hline PD Full & $31.5 \pm 4.3$ & $28.4 \pm 4.7$ & $26.2 \pm 6.9$ & $25.4 \pm 5.6$ & $<0.001 *$ & $<0.001 *$ \\
\hline FAZ Area & $0.21 \pm 0.15$ & $0.17 \pm 0.12$ & $0.17 \pm 0.14$ & $0.19 \pm 0.14$ & $0.002^{*}$ & $0.012 *$ \\
\hline FAZ Perimeter & $2.13 \pm 1.13$ & $1.80 \pm 0.78$ & $1.82 \pm 0.95$ & $1.95 \pm 0.95$ & $0.004 *$ & $0.024 *$ \\
\hline FAZ Circularity & $0.56 \pm 0.14$ & $0.61 \pm 0.10$ & $0.61 \pm 0.15$ & $0.57 \pm 0.15$ & 0.15 & 0.31 \\
\hline
\end{tabular}

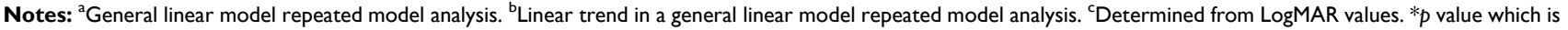
statistically significant.

Abbreviations: CMT, central macular thickness; VD, vascular density; PD, perfusion density; FAZ, foveal avascular zone.

Table 2 Pearson Correlation Between LogMAR, CMT, Area and Perimeter of the FAZ on One Hand and VD and PD on the Other Hand

\begin{tabular}{|c|c|c|c|c|c|c|c|c|}
\hline & \multicolumn{2}{|c|}{ LogMAR } & \multicolumn{2}{|l|}{ CMT } & \multicolumn{2}{|c|}{ FAZ Area } & \multicolumn{2}{|c|}{ FAZ Perimeter } \\
\hline & $r$ & $p$ & $\boldsymbol{r}$ & $p$ & $r$ & $p$ & $r$ & $p$ \\
\hline LogMAR & & & 0.57 & $<0.001 *$ & 0.089 & 0.42 & 0.090 & 0.42 \\
\hline CMT & 0.57 & $<0.001^{*}$ & & & 0.13 & 0.22 & 0.14 & 0.22 \\
\hline VD Central & 0.42 & $<0.001 *$ & 0.60 & $<0.001^{*}$ & 0.33 & $0.003 *$ & 0.30 & $0.005^{*}$ \\
\hline VD Inner & 0.22 & $0.030 *$ & 0.30 & $0.003^{*}$ & 0.45 & $<0.001 *$ & 0.44 & $<0.001 *$ \\
\hline VD Outer & 0.075 & 0.47 & 0.27 & $0.008^{*}$ & 0.57 & $<0.001 *$ & 0.55 & $<0.001^{*}$ \\
\hline VD Full & 0.15 & 0.15 & 0.31 & $0.002^{*}$ & 0.56 & $<0.001 *$ & 0.54 & $<0.001 *$ \\
\hline PD Central & 0.49 & $<0.00 I^{*}$ & 0.57 & $<0.00 I^{*}$ & 0.31 & $0.005^{*}$ & 0.28 & $0.009 *$ \\
\hline PD Inner & 0.19 & 0.060 & 0.29 & $0.004^{*}$ & 0.48 & $<0.001^{*}$ & 0.47 & $<0.001 *$ \\
\hline PD Outer & 0.029 & 0.78 & 0.21 & $0.042^{*}$ & 0.58 & $<0.001 *$ & 0.57 & $<0.00 I^{*}$ \\
\hline PD Full & 0.095 & 0.36 & 0.25 & $0.013^{*}$ & 0.59 & $<0.001 *$ & 0.57 & $<0.001^{*}$ \\
\hline FAZ Area & 0.089 & 0.42 & 0.13 & 0.22 & & & 0.97 & $<0.00 I^{*}$ \\
\hline FAZ Perimeter & 0.090 & 0.42 & 0.14 & 0.22 & 0.97 & $<0.00 I^{*}$ & & \\
\hline FAZ Circularity & -0.11 & 0.32 & -0.10 & 0.36 & -0.62 & $<0.001 *$ & -0.74 & $<0.001^{*}$ \\
\hline
\end{tabular}

Note: * $p$ value which is statistically significant.

Abbreviations: CMT, central macular thickness; VD, vascular density; PD, perfusion density; FAZ, foveal avascular zone.

et al, it is particularly of interest to note that the VD differences between the eyes with and without macular oedema were not significantly different, suggesting differential axes of regulation and possibly non-VEGFmediated mechanisms at play. ${ }^{22}$

In specific view of the VD measurements following anti-VEGF therapy, current reportage of studies indicates a bi-directional response. ${ }^{23}$ While most of the studies have shown either a neutral or a positive change in $\mathrm{VD}^{14,20,24-26}$ following anti-VEGF injections, a few have also reported a reduction in VD following anti-VEGF. ${ }^{27}$ At the same time Tomita et al reported a lower VD to be a predictor of recurrence of oedema. ${ }^{27}$ The precise nature of the changes in VD as well as its relation to characterizing macular perfusion specifically following anti-VEGF therapy remains unclear, and considerable variability continues to exist, although a few long-term follow-up studies have noted a stable or no change in VD following antiVEGF. $^{28}$ In our study, a significant reduction in VD and PD was seen, which is indicative of progression of 
Table $3 \beta$-Coefficients from a Linear Mixed Model Analysis

\begin{tabular}{|c|c|c|c|c|c|}
\hline & \multirow[t]{2}{*}{ Intercept } & \multicolumn{2}{|l|}{ CMT } & \multicolumn{2}{|c|}{ FAZ Area } \\
\hline & & $\boldsymbol{\beta}$ & $p$ & $\beta$ & $p$ \\
\hline LogMAR & 0.073 & 0.00037 & $0.011^{*}$ & 0.12 & 0.22 \\
\hline VD Central & -3.13 & 0.019 & $<0.001^{*}$ & 5.09 & $0.003 *$ \\
\hline VD Inner & 6.14 & 0.0092 & $0.005^{*}$ & 9.33 & $<0.00 I^{*}$ \\
\hline VD Outer & 8.44 & 0.0046 & $0.04 I^{*}$ & 8.69 & $<0.00 I^{*}$ \\
\hline VD Full & 7.62 & 0.0062 & $0.005^{*}$ & 8.25 & $<0.001^{*}$ \\
\hline PD Central & -6.58 & 0.041 & $<0.001^{*}$ & 11.96 & $0.004^{*}$ \\
\hline PD Inner & $|4.4|$ & 0.021 & $0.009 *$ & 25.31 & $<0.001 *$ \\
\hline PD Outer & 21.07 & 0.0091 & 0.123 & 23.15 & $<0.001^{*}$ \\
\hline PD Full & $|8.8|$ & 0.012 & $0.033^{*}$ & 23.45 & $<0.00 I^{*}$ \\
\hline
\end{tabular}

Note: ${ }^{*} p$ value which is statistically significant.

Abbreviations: CMT, central macular thickness; VD, vascular density; PD, perfusion density; FAZ, foveal avascular zone.

ischemia. At the same time, we noted a simultaneous reduction in FAZ size which is paradoxical, as it is indicative of improvement in macular perfusion. Nonetheless, a reduction in FAZ size can also occur because of a decrease in manual displacement of retinal capillaries secondary to improvement in macular oedema. This reduction in macular fluid or the central CMT was evidently seen in our series. Hence, we postulate that the primary reason for a reduction in FAZ size as seen in our study is due to a concurrent decrease in capillary displacement secondary to resolution of intraretinal oedema, rather than an improvement in macular perfusion. The FAZ circularity too remained unaltered during the three-month period, indicating no change in the configuration of the FAZ capillary margins. This outcome also corroborates the mechanical displacement theory of changes seen in FAZ size rather than an ischemic cause. Nonetheless, studies continue to report differential responses of the retinal microvasculature measurements in the wake of DME, even with OCTA. Although comparability amongst the studies is noted to be presently very low, more harmonization in the future could aid better and meaningful comparisons.

It appears that the macular vessel density in DME has a multifactorial origin, and a role of the pericyte and its modelling of the autoregulatory function of retinal capillaries could be a supplementary axis of dysfunction in addition to the VEGF-mediated vascular changes. While the loss of retinal pericytes has been well established in the breakdown of inner BRB, the precise mechanisms are not very clear yet. ${ }^{29}$ Also, the neurovascular coupling in the retinal perfusion linked to active relaxation of the capillaries is understood to be regulated in part by the coupling of the endothelial cell-pericyte interaction. ${ }^{30}$ It needs to be seen if pericyte dysfunction represents a residual effect following anti-VEGF therapy.

Different studies have chosen different scan protocols. Ho et al showed that there was clinical utility for both the higher scan resolution obtainable with a smaller $(3 \mathrm{~mm} \times 3 \mathrm{~mm})$ scan, as well as the larger field of view obtained with a larger $(6 \mathrm{~mm} \times 6 \mathrm{~mm})$ OCTA scan protocol in assessing patients with diabetic retinopathy. The more diffuse details of capillary nonperfusion and presence of microaneurysms were better delineated with the $6 \mathrm{~mm} \times 6 \mathrm{~mm}$ scans, though FAZ measurements were more reliable with $3 \mathrm{~mm} \times 3 \mathrm{~mm} .{ }^{31}$ Excellent results were found for FAZ area interrater reliability for each single plexus in the same only for all retina and superficial vascular layers in smaller angiocubes (ie, $3 \times 3 \mathrm{~mm}$ and $6 \times 6 \mathrm{~mm}$ ). However, less but still satisfactory reliability was disclosed for $12 \times 12-\mathrm{mm}$ angiocube at the same plexuses. ${ }^{32}$ We chose the $6 \mathrm{~mm}$ $\times 6 \mathrm{~mm}$ scan protocol as a balance between $3 \mathrm{~mm}$ and $12 \mathrm{~mm}$ angiocubes, and it delineated the capillary nonperfusion details better.

With better adoption of OCTA, further studies are required to understand the temporospatial responses in the retinal microvasculature and cone mosaic to antiVEGF therapy and its resultant effect in improving the visual acuity.

It must be noted that this study has some limitations. We did not control for factors such as axial length, stage of DR and age, which may affect the quantitative OCTA data. While the OCTA method has been noted to be a very reliable and accurate technique to delineate the retinal thickness and FAZ, measures related to the vessel and perfusion density are yet to be perfected, and depend on the availability of suitable software algorithms, the characterization of the effect of oedema and underlying pathology. Also, the current measurements were limited to the measuring the superficial capillary plexus (SCP) alone, as routine deep capillary plexus (DCP) measurements on OCTA are not bereft with poor visualization and low reliability especially in the patients with significant oedema. Even though few earlier studies have noted a differential predictability of the DCP measures on changes in treatment outcomes, it had to be done in retrospect instead of the current method to ascertain OCTA parameters prospectively. ${ }^{7}$ In presence of significant oedema, there is a possibility of artefacts impacting the 
accurate measurements of SCP. To overcome this potential limitation, a single trained reader manually segmented the OCTA sections. Also, by utilizing only the scans with SSI $>6$ for analysis, we negated the likelihood of inaccurate measurements. Finally, a smaller sample size with short follow-up period is a drawback of the study. Studies with larger sample size and longer follow-up duration would provide better insight into the role of anti-VEGF agents in modifying macular vascularity in DME on OCTA. Furthermore, additional understanding and comparative evaluation of these parameters in treatment-naïve and previously treated eyes, which was lacking in our study due to small sample size, would be recommended.

The current study provides the first real-world data regarding the morphometric parameters on OCTA in an Indian scenario. The current study would be invaluable to assess and compare these OCTA parameters with other ethnic populations to look for any variation in treatment response. Moreover, very limited data exists regarding these OCTA changes in DME management. Limited studies have mostly utilized PRN regimen and other agents such as aflibercept and bevacizumab. ${ }^{20,21,33}$ Hence by utilizing a fixed dose regimen of ranibizumab we aim to provide valuable real-world evidence to add to the existing literature. Also, we report a novel finding of reduction in FAZ size with a paradoxical simultaneous decrease in VD and $\mathrm{CD}$ in response to anti-VEGF therapy in DME in the short term.

Finally, the current study demonstrated, with use of intravitreal anti-VEGF therapy, that there is significant improvement in visual functions in patients with DME at three months. A simultaneous reduction in CMT was the most reliable predictor for visual response. On OCTA, a reduction in FAZ was noted which can be hypothesized to be due to a concurrent reduction in manual displacement of the FAZ due to resolution of diabetic macular oedema.

\section{Future Directions}

Based on the current study results we propose that OCTA (SCP) markers in a routine clinical scenario could play a good role in measuring and establishing early progress to anti-VEGF therapy. This could help individualize the standard of care in individuals with retinopathy and macular oedema. More studies are needed to address the reliability and characterization of temporospatial changes in the OCTA in the long term.

\section{Code Availability (Software Application or Custom Code)}

SPSS code used for statistical analysis will be made available to anyone who wants access.

\section{Data Sharing Statement}

Datasheet with anonymized patient data will be made available to anyone who wants access.

\section{Ethics Approval}

The ethics committee of Rajan Eye Care approved this study.

\section{Consent to Participate}

Written informed consent was taken from all patients.

\section{Author Contributions}

All authors made a significant contribution to the work reported, whether that is in the conception, study design, execution, acquisition of data, analysis and interpretation, or in all these areas; took part in drafting, revising or critically reviewing the article; gave final approval of the version to be published; have agreed on the journal to which the article has been submitted; and agree to be accountable for all aspects of the work.

\section{Funding}

There is no funding to report.

\section{Disclosure}

CAB Webers report grants and personal fees from Alcon/ Novartis and Santen and personal fees from Thea Pharma, outside the submitted work. The authors report no other potential conflicts of interest for this work.

\section{References}

1. Rajalakshmi R, Behera UC, Bhattacharjee H, et al. Spectrum of eye disorders in diabetes (SPEED) in India. Report \# 2. Diabetic retinopathy and risk factors for sight threatening diabetic retinopathy in people with type 2 diabetes in India. Indian J Ophthalmol. 2020;68 (13):21. doi:10.4103/ijo.IJO_21_19

2. Raman R, Vaitheeswaran K, Vinita K, Sharma T. Is prevalence of retinopathy related to the age of onset of diabetes? Sankara nethralaya diabetic retinopathy epidemiology and molecular genetic report no. 5 . Ophthalmic Res. 2010. doi:10.1159/000314720

3. Romero-Aroca P, Baget-Bernaldiz M, Pareja-Rios A, Lopez-Galvez M, Navarro-Gil R, Verges R. Diabetic macular edema pathophysiology: vasogenic versus inflammatory. J Diabetes Res. 2016;2016:1-17. doi: $10.1155 / 2016 / 2156273$

4. Cunha-Vaz J, Bernardes R, Lobo C. Blood-retinal barrier. Eur J Ophthalmol. 2011;21(6_suppl):3-9. doi:10.5301/EJO.2010.6049 
5. Virgili G, Parravano M, Evans JR, Gordon I, Lucenteforte E. Antivascular endothelial growth factor for diabetic macular oedema: a network meta-analysis. Cochrane Database Syst Rev. 2018. doi:10.1002/14651858.CD007419.pub6

6. Kwan CC, Fawzi AA. Imaging and biomarkers in diabetic macular edema and diabetic retinopathy. Curr Diab Rep. 2019;19(10). doi:10.1007/s11892-019-1226-2

7. Lee J, Moon BG, Cho AR, Yoon YH. Optical coherence tomography angiography of DME and its association with Anti-VEGF treatment response. Ophthalmology. 2016;123(11):2368-2375. doi:10.1016/j. ophtha.2016.07.010

8. Sugimoto M, Ichio A, Mochida D, et al. Multiple effects of intravitreal aflibercept on microvascular regression in eyes with diabetic macular edema. Ophthalmol Retina. 2019;3(12):1067-1075. doi:10.1016/j.oret.2019.06.005

9. Choi KE, Yun C, Cha J, Kim SW. OCT angiography features associated with macular edema recurrence after intravitreal bevacizumab treatment in branch retinal vein occlusion. Sci Rep. 2019. doi:10.1038/s41598-019-50637-8

10. Agarwal A, Soliman MK, Sepah YJ, Do DV, Nguyen QD. Diabetic retinopathy: variations in patient therapeutic outcomes and pharmacogenomics. Pharmgenomics Pers Med. 2014;7:399-409. doi:10.2147/PGPM.S52821

11. Sadda SR, Campbell J, Dugel PU, et al. Relationship between duration and extent of oedema and visual acuity outcome with ranibizumab in diabetic macular oedema: a post hoc analysis of Protocol I data. Eye. 2019. doi:10.1038/s41433-019-0522-z

12. Enders C, Baeuerle F, Lang GE, et al. Comparison between findings in optical coherence tomography angiography and in fluorescein angiography in patients with diabetic retinopathy. Ophthalmologica. 2020;243(1):21-26. doi:10.1159/000499114

13. Moein HR, Novais EA, Rebhun CB, et al. Optical coherence tomography angiography to detect macular capillary ischemia in patients with inner retinal changes after resolved diabetic macular edema. Retina. 2018;38(12):2277-2284. doi:10.1097/IAE.0000000000001902

14. Dastiridou A, Karathanou K, Riga P, et al. OCT angiography study of the macula in patients with diabetic macular edema treated with intravitreal aflibercept. Ocul Immunol Inflamm. 2019. doi:10.1080/ 09273948.2019.1704028

15. Kozulin P, Natoli R, Bumsted O’Brien KM, Madigan MC, Provis JM. Differential expression of anti-angiogenic factors and guidance genes in the developing macula. Mol Vis. 2009.

16. Kozulin P, Provis JM. Differential gene expression in the developing human macula: microarray analysis using rare tissue samples. J Ocul Biol Dis Infor. 2009;2(4):176-189. doi:10.1007/s12177-009-9039-1

17. Dabir S, Mangalesh S, Kumar KA, Kummelil MK, Sinha Roy A, Shetty R. Variations in the cone packing density with eccentricity in emmetropes. Eye. 2014;28(12):1488-1493. doi:10.1038/eye.2014. 229

18. Lei J, Durbin MK, Shi Y, et al. Repeatability and reproducibility of superficial macular retinal vessel density measurements using optical coherence tomography angiography en face images. JAMA Ophthalmol. 2017;135(10):1092. doi:10.1001/jamaophthalmol.20 17.3431

19. Durbin MK, An L, Shemonski ND, et al. Quantification of retinal microvascular density in optical coherence tomographic angiography images in diabetic retinopathy. JAMA Ophthalmol. 2017;135(4):370. doi:10.1001/jamaophthalmol.2017.0080
20. Ghasemi Falavarjani K, Iafe NA, Hubschman JP, Tsui I, Sadda SR, Sarraf D. Optical coherence tomography angiography analysis of the foveal avascular zone and macular vessel density after anti-VEGF therapy in eyes with diabetic macular edema and retinal vein occlusion. Investig Ophthalmol Vis Sci. 2017;58(1):30. doi:10.1167/iovs.16-20579

21. Hsieh YT, Alam MN, Le D, et al. OCT angiography biomarkers for predicting visual outcomes after ranibizumab treatment for diabetic macular edema. Ophthalmol Retina. 2019;3(10):826-834. doi:10.1016/j.oret.2019.04.027

22. AttaAllah HR, Mohamed AAM, Ali MA. Macular vessels density in diabetic retinopathy: quantitative assessment using optical coherence tomography angiography. Int Ophthalmol. 2019;39(8):1845-1859. doi:10.1007/s10792-018-1013-0

23. Wai KM, Singh RP. Changes in macular and peripheral perfusion following anti-vascular endothelial growth factor treatment for patients with diabetic retinopathy. Am J Ophthalmic Clin Trials. 2019;2:4. doi:10.25259/ajoct-3-2019

24. Sorour OA, Sabrosa AS, Yasin Alibhai A, et al. Optical coherence tomography angiography analysis of macular vessel density before and after anti-VEGF therapy in eyes with diabetic retinopathy. Int Ophthalmol. 2019;39(10):2361-2371. doi:10.1007/s10792-019-01076-x

25. Fan W, Uji A, Wang K, et al. Severity of diabetic macular edema correlates with retinal vascular bed area on ultra-wide field fluorescein angiography. Retina. 2019. doi:10.1097/iae.0000000000002579

26. Michaelides M, Fraser-Bell S, Hamilton R, et al. Macular perfusion determined by fundus fluorescein angiography at the 4-month time point in a prospective randomized trial of intravitreal bevacizumab or laser therapy in the management of diabetic macular edema (bolt study): report 1. Retina. 2010;30(5):781-786. doi:10.1097/IAE.0b013e3181d2f145

27. Tomita R, Iwase T, Goto K, Yamamoto K, Ra E, Terasaki H. Correlation between macular vessel density and number of intravitreal anti-VEGF agents for macular edema associated with branch retinal vein occlusion. Sci Rep. 2019;9(1). doi:10.1038/s41598-019-52732-2

28. Karst SG, Deak GG, Gerendas BS, et al. Association of changes in macular perfusion with ranibizumab treatment for diabetic macular edema. JAMA Ophthalmol. 2018;136(4):315. doi:10.1001/ jamaophthalmol.2017.6135

29. Klaassen I, Van Noorden CJF, Schlingemann RO. Molecular basis of the inner blood-retinal barrier and its breakdown in diabetic macular edema and other pathological conditions. Prog Retin Eye Res. 2013;34:19-48. doi:10.1016/j.preteyeres.2013.02.001

30. Trost A, Lange S, Schroedl F, et al. Brain and retinal pericytes: origin, function and role. Front Cell Neurosci. 2016;10. doi:10.3389/fncel.2016.00020

31. Ho J, Dans K, You Q, et al. Comparison of $3 \mathrm{~mm} \mathrm{X} 3 \mathrm{~mm}$ versus $6 \mathrm{~mm}$ X $6 \mathrm{~mm}$ optical coherence tomography angiography scan sizes in the evaluation of non proliferative diabetic retinopathy. Retina. 2019;39:259-264. doi:10.1097/IAE.0000000000001951

32. Rabiolo A, Gelormini F, Marchese A, et al. Macular perfusion parameters in different angiocube sizes: does the size matter in quantitative optical coherence tomography angiography? Invest Ophthalmol Vis Sci. 2018;59:231-237. doi:10.1167/iovs.17-22359

33. Elnahry AG, Abdel-Kader AA, Raafat KA, Elrakhawy K. Evaluation of changes in macular perfusion detected by optical coherence tomography angiography following 3 intravitreal monthly bevacizumab injections for diabetic macular edema in the IMPACT study. J Ophthalmol. 2020;2020:5814165. doi:10.1155/2020/5814165 


\section{Publish your work in this journal}

Clinical Ophthalmology is an international, peer-reviewed journal covering all subspecialties within ophthalmology. Key topics include: Optometry; Visual science; Pharmacology and drug therapy in eye diseases; Basic Sciences; Primary and Secondary eye care; Patient Safety and Quality of Care Improvements. This journal is indexed on PubMed
Central and CAS, and is the official journal of The Society of Clinical Ophthalmology (SCO). The manuscript management system is completely online and includes a very quick and fair peer-review system, which is all easy to use. Visit http://www.dovepress.com/ testimonials.php to read real quotes from published authors.

Submit your manuscript here: https://www.dovepress.com/clinical-ophthalmology-journal 